\title{
Dynamical and Microphysical Constraints on Accreting Magnetospheric Clouds in Active Galactic Nuclei
}

\author{
Zdenka Kuncic ${ }^{1}$ \\ ANU Astrophysical Theory Centre, Australian National University, \\ Canberra ACT 0200, Australia
}

\begin{abstract}
The range of microphysical and dynamical timescales at the centres of Active Galactic Nuclei (AGN) is sufficiently wide to permit multiphase structure. In particular, very dense, cool clouds can coexist with a hot, magnetically-dominated plasma. The strong dynamical forces in this central magnetosphere can give rise to clouds so small that microphysical processes determine whether they can survive for long enough to produce potentially observable spectral signatures due to thermal reprocessing. Specific physical constraints on the scale sizes of such reprocessing clouds are examined. It is found that there exists a parameter subspace in the extreme high density regime for which gas can sustain cool temperatures whilst maintaining pressure equilibrium with the ambient magnetosphere.
\end{abstract}

\section{Introduction}

The strong gravitational, radiation and magnetic fields in the central regions of AGN maintain any accreting gas in a state of ongoing dynamical activity. The relevant microphysical timescales (e.g. thermalization, cooling) can, however, be extremely short compared to the global dynamical timescale (Rees 1984). This suggests that an instantaneous 'snapshot' could reveal the existence of small, quasi-equilibrium structures that are capable of producing spectral signatures.

A picture in which cool clumps of material are embedded in much hotter and more tenuous gas has been successful in accounting for a variety of thermal reprocessing signatures in AGN spectra (e.g. broad UV emission and absorption lines, 'warm absorber' soft X-ray edges) which are inferred to arise from gas residing at distances $(.01-1)$ pc from the central source (e.g. Davidson 1972, Netzer 1993).

Such a multiphase picture may also apply to the central source itself, where cool clouds will be many orders of magnitude denser than the reprocessing gas residing further out and where the strong confining forces can give rise to cloud sizes which are extremely small by most astrophysical standards (e.g. Rees 1987, Celotti et al. 1992, Barvainis 1993, Kuncic et al. 1996). These unusual physical conditions warrant further investigation: can such small-scale, dense clouds survive in the central magnetospheres of AGN long enough to be detected through thermal reprocessing signatures in the observed spectra?

${ }^{1}$ This work was undertaken and completed at the Institute of Astronomy, Cambridge UK. 


\section{Dynamical constraints - radiative and hydrostatic equilibrium}

In order for clouds to cool to a temperature $T_{\mathrm{e}}$ before falling into the strong gravitational well at the centre of an AGN, the timescale $t_{\mathrm{rad}}$ for radiative cooling must be much shorter than the dynamical timescale. This requires electron densities (Kuncic et al. 1996)

$$
n_{\mathrm{e}} \gg 10^{16}\left(\frac{T_{\mathrm{e}}}{T_{\mathrm{vir}}}\right)^{1 / 2}\left(\frac{r}{r_{\mathrm{g}}}\right)^{-2} M_{7}^{-1} \mathrm{~cm}^{-3},
$$

where $T_{\text {vir }}=m_{\mathrm{p}} v_{\mathrm{ff}}^{2} / k$ is the virial temperature corresponding to a free-fall speed $v_{\mathrm{ff}}=c\left(r / r_{\mathrm{g}}\right)^{-1 / 2}$ and $r_{\mathrm{g}}=G M / c^{2} \approx\left(1.5 \times 10^{12}\right) M_{7} \mathrm{~cm}$ is the gravitational radius of a central mass $M=10^{7} M_{7} M_{\odot}$.

At the same time, the dense clouds can only be confined by an ambient magnetosphere if the sound-crossing time is shorter than $t_{\mathrm{ff}}=r / v_{\mathrm{ff}}$. This implies an upper bound on the characteristic thickness $R_{\mathrm{cld}}$ of a cloud,

$$
R_{\mathrm{cld}}<t_{\mathrm{ff}} v_{\mathrm{s}}
$$

where the total internal sound speed is given by $v_{\mathbf{s}}^{2}=\left(2 k T_{\mathrm{e}} / m_{\mathrm{p}}\right)(1+1 / \beta)(\beta$ is the usual plasma beta parameter).

The pressure scaleheight Dense clouds can feel a strong radiation pressure, which gives rise to an effective gravity term, $g^{*}$, in the standard equation of magnetohydrostatic equilibrium,

$$
n_{\mathrm{e}} m_{\mathrm{p}} g_{i}\left(1-\frac{g^{*}}{g}\right)=\nabla_{i}\left(n_{\mathrm{e}} k T_{\mathrm{e}}+\frac{B^{2}}{8 \pi}\right)-\nabla_{j}\left(\frac{B_{j} B_{i}}{4 \pi}\right) .
$$

Very small scaleheights can be achieved, with $h_{\text {cld }}$ reduced by a factor $g^{*} / g \gg$ 1 , by the compressive action of radiation forces against the magnetic stresses which confine dense clouds (Celotti et al. 1992, Kuncic et al. 1996). Such clouds are unlikely to exist as infinitessimally thin structures, however, since their survival is then threatened by the microphysical processes which act to disrupt multiphase structure.

\section{Microphysical constraints - thermal conduction}

The high-energy spectra of most AGN imply mildly relativistic temperatures for the emitting plasma (see Zdziarski et al. 1995). The high thermal conductivity of such an ambient hot plasma poses the most serious threat to the continued existence of small-scale, cool clouds embedded within it (see Kuncic et al. 1996 and references therein).

The thermal diffusion coefficient is given by $D_{\mathrm{T}} \approx \lambda_{\mathrm{T}}\left(k T_{\mathrm{e}} / m_{\mathrm{e}}\right)^{1 / 2}$, where the effective path length for collisions between the hot electrons and the denser cloud particles is

$$
\lambda_{\mathrm{T}} \sim\left(3 \times 10^{3}\right)\left(\frac{n_{\mathrm{cld}}}{10^{18} \mathrm{~cm}^{-3}}\right)^{-1}\left(\frac{T_{\text {hot }}}{10^{9} \mathrm{~K}}\right)^{2} \mathrm{~cm} .
$$


The depth to which hot electrons can diffuse into cool clouds during a timescale $\Delta t$ (in seconds) is typically

$$
d_{\mathrm{T}} \sim \sqrt{D_{\mathrm{T}} \Delta t} \sim\left(6 \times 10^{6}\right)\left(\frac{n_{\mathrm{cld}}}{10^{18} \mathrm{~cm}^{-3}}\right)^{-1 / 2}\left(\frac{T_{\mathrm{hot}}}{10^{9} \mathrm{~K}}\right)^{5 / 4}(\Delta t)^{1 / 2} \mathrm{~cm} .
$$

Diffusion across magnetic field lines is negligible by comparison.

Clouds with $R_{\text {cld }}>\lambda_{\mathrm{T}}$ are susceptible to evaporation, unless their densities are higher than the critical density

$$
\frac{n_{\text {crit }}}{n_{\text {hot }}} \sim\left(2 \times 10^{6}\right)\left(\frac{T_{\text {cld }}}{10^{5} \mathrm{~K}}\right)^{-1 / 2}\left(\frac{T_{\text {hot }}}{10^{9} \mathrm{~K}}\right)^{-1 / 2}
$$

at which the additional heat input from hot electrons can be efficiently radiated away. When $n_{\text {cld }}>n_{\text {crit }}$, it is possible for clouds to remain relatively cool whilst threaded by field lines to which hot electrons are also tied. This holds even when $R_{\mathrm{cld}}<\lambda_{\mathrm{T}}$, since although the conducting electrons are then unimpeded by collisions, they can impart energy to the clouds by dynamical friction instead.

Pair Plasma Effects Thermal conduction by electron-positron $\left(e^{+} e^{-}\right)$pairs poses a particularly important threat to the survival of cool, dense clouds at the centres of AGN. This is because once the compactness parameter $l \equiv \sigma_{\mathrm{T}} L / m_{\mathrm{e}} c^{3} r$ satisfies $f_{\gamma} l>4 \pi$, where $f_{\gamma}$ is the fraction of primary luminosity that is emitted above $1 \mathrm{MeV}$, the encounters between $\gamma$-ray photons which produce pairs can occur anywhere - even within clouds themselves. Pairs created 'in situ' can provide a roughly uniform heat input before they annihilate. There is a corresponding minimum cloud density at which this extra heat input can be balanced by radiative cooling (see Kuncic et al. 1996 for details).

\section{Results and discussion}

Fig. 1 shows the parameter space ( $n_{\text {cld }}$ vs. $R_{\text {cld }}$ ) diagram for clouds with a constant temperature $T_{\text {cld }}=10^{5} \mathrm{~K}$ in pressure equilibrium with a diffuse, magnetized intercloud plasma with a constant temperature $T_{\text {hot }}=10^{9} \mathrm{~K}$. The source region has a size $r=10^{13} \mathrm{~cm} \sim 10 r_{\mathrm{g}} M_{7}^{-1}$ from which emerges a total luminosity $L=10^{44} \mathrm{erg} \cdot \mathrm{s}^{-1}$. These quantities are representative of current ideas for the central regions of most AGN (e.g. Zdziarski 1995). The plot shows regions restricted by the following physical constraints: the maximum thickness, $t_{\mathrm{ff}} v_{\mathbf{s}}$, for pressure support; the pressure scaleheight, $h$; the thermal diffusion depth $d_{T}$ for various timescales; and the path length $\lambda_{T}$ over which collisions are effective. Also shown are the diffusion depth $d_{\mathrm{T}, \perp}$ transverse to magnetic field lines and the diffusion depth $d_{\text {pair }}$ for thermal pairs, which is the same as $d_{\mathrm{T}}$, but calculated for an annihilation timescale $t_{\mathrm{ann}} \sim(r / c)(l / 4 \pi)^{-1 / 2}$. The cross-hatches identify the subspace for which clouds cannot be confined by the ambient magnetosphere.

The darkest shaded region in the plot indicates where radiative cooling prevails; any additional heating due to thermal conduction can be efficiently radiated away, so that cool gas always prevails. It is this dense gas which is capable of producing thermal reprocessing signatures in the spectra. The next 


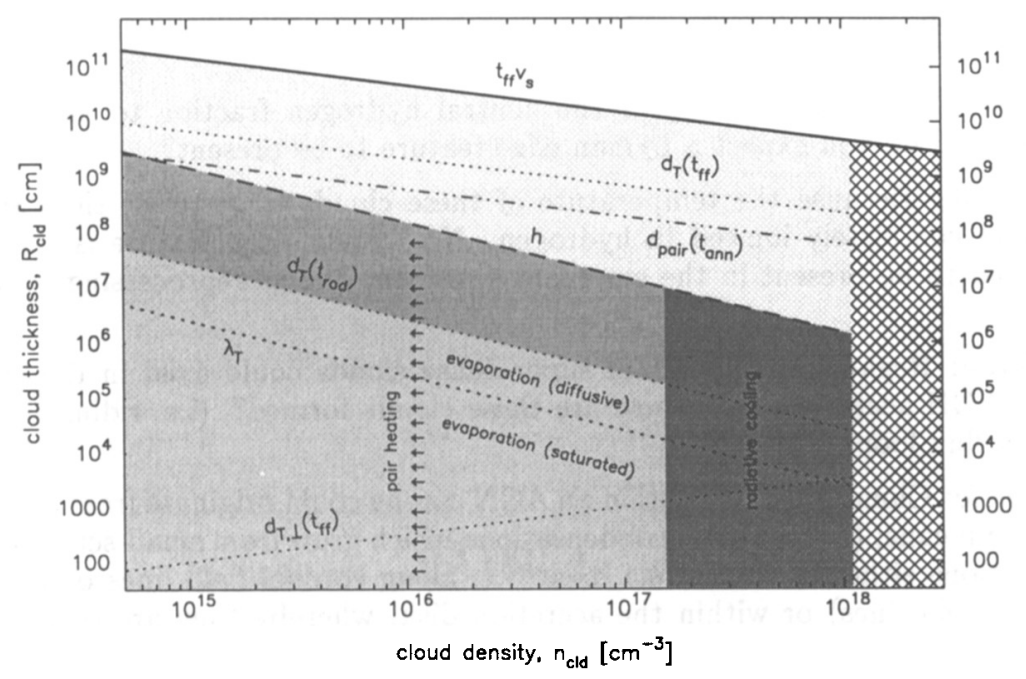

Figure 1. Parameter space for clouds at the centres of AGN, indicating densities and sizes at which clouds can survive against dynamical and microphysical processes. All labels are defined in the text.

darkest shaded region in the plot indicates where clouds threaded by the same field lines to which hot electrons are also tied can survive the effects of conduction for timescales $z t_{\text {rad }}$ which may be sufficient to produce spectral signatures. Such clouds are unlikely to be able to survive on timescales approaching $t_{\mathrm{ff}}$, since $d_{\mathrm{T}}$ eventually becomes comparable to $h$ and evaporation is then unavoidable. Moreover, in the presence of an effective gravity (due to radiation pressure, for instance), $h$ can be substantially reduced and hot electrons can then readily diffuse into dense clouds on timescales shorter than $t_{\mathrm{ff}}$. Thus, such clouds can only survive if coupled to separate field lines or as self-contained structures, decoupled from the magnetosphere.

\section{References}

Barvainis, R., 1993, ApJ, 412, 513

Celotti, A., Fabian, A. C. \& Rees, M. J. 1992, MNRAS, 255, 419

Davidson, K. 1972, ApJ, 171, 213

Kuncic, Z., Blackman, E. G. \& Rees, M. J. 1996, MNRAS(in press)

Netzer, H. 1993, ApJ, 411, 594

Rees, M. J. 1984, ARA\&A, 22, 477

Rees, M. J. 1987, MNRAS, 228, 47p

Zdziarski, A. A., Johnson, W. N., Done, C., Smith, D. \& McNaron-Brown, K., 1995, ApJ, 438, L63 


\section{Discussion}

O. Blaes: What do you expect the neutral hydrogen fraction to be in these clouds, and do you expect a Lyman edge feature to be present?

Z. Kuncic: Because the temperature of these clouds is $\geq 10^{5} \mathrm{~K}$, they are essentially completely ionised in hydrogen. No Lyman edge feature is therefore expected to be present in the emergent spectrum due to reprocessing by these clouds.

M. Owen: Regardless of whether super-dense clouds could exist in the corona above AGN accretion disks, how are these clouds formed? (i.e. riding up field lines in flux loops, etc).

Z. Kuncic: Clouds confined within an AGN corona could originate from at least 3 possible means; (i) from local condensations which grow from small-scale density fluctuations either in the corona itself (i.e. along coronal field lines or between tangled field lines) or within the accretion disk, whereby they are removed by the field lines, (ii) from ejections of diamagnetic plasmoids formed in the $\mathrm{AD}$ from small-scale reconnection events and (iii) from small amounts of accreting matter that does not manage to fall into the $A D$ before being caught in the corona by magnetic fields. 